\title{
STUDY OF INTERPLANETARY DISTURBANCES USING IPS
}

\author{
S. ANANTHAKRISHNAN \\ National Centre for Radio Astrophysics \\ Tata Institute of Fundamental Research \\ Poona University Campus \\ Pune 411007 , India
}

\begin{abstract}
The usefulness of Interplanetary Scintillation (IPS) data, which provide solar wind velocities $(\mathrm{V})$ and relative scintillation indices $(\mathrm{g})$, for predicting interplanetary disturbances is examined. Analysis of two years of g-map data from Cambridge shows that atleast two IPS stations are required for more definitive identification of events. Campaigns were made in April-May 1992 to map predicted solar wind disturbances simultaneously from two widely separated telescopes at Cambridge and Ooty. These show that apriori knowledge of strong flare activity helps in detecting scintillation enhancement. On the other hand, other events have been observed at Ooty, which show that both flares and coronal holes may be responsible for producing interplanetary disturbances, and hence it is premature to identify any one type of solar event as the sole cause of the disturbances.
\end{abstract}

\section{Introduction}

Radio waves from distant radio sources are scattered by the variations in the electron density in the turbulent interplanetary medium (solar wind) causing intensity fluctuations on the ground. This gives rise to interplanetary scintillation (IPS). A guestimate of the electron density in the IPM can be made by assuming that the density fluctuations, $\Delta n$ are related to the mean density $n$ and by estimating $\Delta n$ by using the scintillation index $m$ ( $r m s$ intensity fluctuations $\Delta S$ mean source intensity $I$ ) which is proportional to $\Delta n$. The intensity scintillations can also be characterised by taking their power spectra. Manoharan and Ananthakrishnan (1990) showed that the power spectra of the electron density fluctuations are well modelled by a power law of the form $q^{-\alpha}$. Using a 5 parameter model they fitted the temporal spectra and estimated the solar wind velocities using a large number of extragalactic radio sources. These single station velocities agree well with the three station velocities estimated by the Nagoya group. During these Ooty observations it was seen that whenever there are solar wind transients, the density as well as velocity derived from IPS tend to increase. The question naturally arises, as to whether the IPS method can be used to detect interplanetary disturbances (IPD's) on a routine basis and whether such detections can be related to events on the Sun and be used to forecast geomagnetic disturbances. We have achieved limited success in answering this 
question by using the results of extensive IPS observations from both the Cambridge transit array $\left(36000 \mathrm{~m}^{2}\right)$ at $80 \mathrm{MHz}$ and the fully steerable Ooty Radio Telescope $\left(15000 \mathrm{~m}^{2}\right)$ at $327 \mathrm{MHz}$.

\section{The Observations and Results}

All sky scintillation maps have been made by the Cambridge IPS group at $81.5 \mathrm{MHz}$ by using the relative scintillation index $g$ which is defined as $g=m_{\text {date }} /\langle m\rangle=\Delta S_{\text {date }} /\left\langle\Delta S_{\epsilon}\right\rangle$. Thus g-values are the ratios of the $r m s$ value of the observed scintillation to the average value expected at the same solar elongation angle, $\epsilon$. A detailed study of two years of these g-maps for the period March 1990 through December 1992 was made by Leinbach, Ananthakrishnan and Detman (1994). They concluded that single station gmaps did not provide a useful tool for forecasting due to a variety of reasons, the most important being the difficulty in identifying IPS events and then interpreting them in terms of solar events as well as geoeffective solar wind disturbances. However, the g-maps do show the presence of quasi steady corotating solar wind structures.

As a different strategy we decided to follow a predictive mode, in which using real time observation of solar events, a Shock Time of Arrival (STOA) model (Dryer and Smart, 1984) was used to make a global estimate of the shock propagation. Two large solar flare events took place in the NOAA region 7154 at $\mathrm{S} 23^{\circ} \mathrm{E} 48^{\circ}$ on May 7 and 8, 1992 and Type II shocks were detected. Increases of scintillation indices and velocities were predicted to take place several hours later in the interplanetary medium. These were detected in the g-maps of Cambridge and were also detected by the steerable Ooty telescope by observing the IPS of an appropriate set of spatially distributed compact extragalactic radio sources as a 'picket fence'. The consistency between the g-values from these two stations appear to confirm the predicted shock trajectories and support a simple physical model of propagating shock structures. More details of these observations can be found in Manoharan et al. (1995). This two-station experiment has shown that multiple observatories can reduce ambiguities in the IPS data and improve the chances of IPD detection. As a second instance of the use of prediction based observations, scintillation enhancements from two flare generated shock fronts, which occured on October 30 and 31, 1992 were observed by the Ooty telescope using a grid of compact radio sources (Janardhan et al., 1995, preprint). Solar wind velocities were estimated and two travelling IPD's were detected. These disturbances were traced back to the Sun. Unlike in the previous case, the activity on the Sun on these two days involved flares from NOAA region 7321 , filament eruption as well as a nearby rapidly evolving coronal hole (Fig. 1). However, no data was available which could indicate 


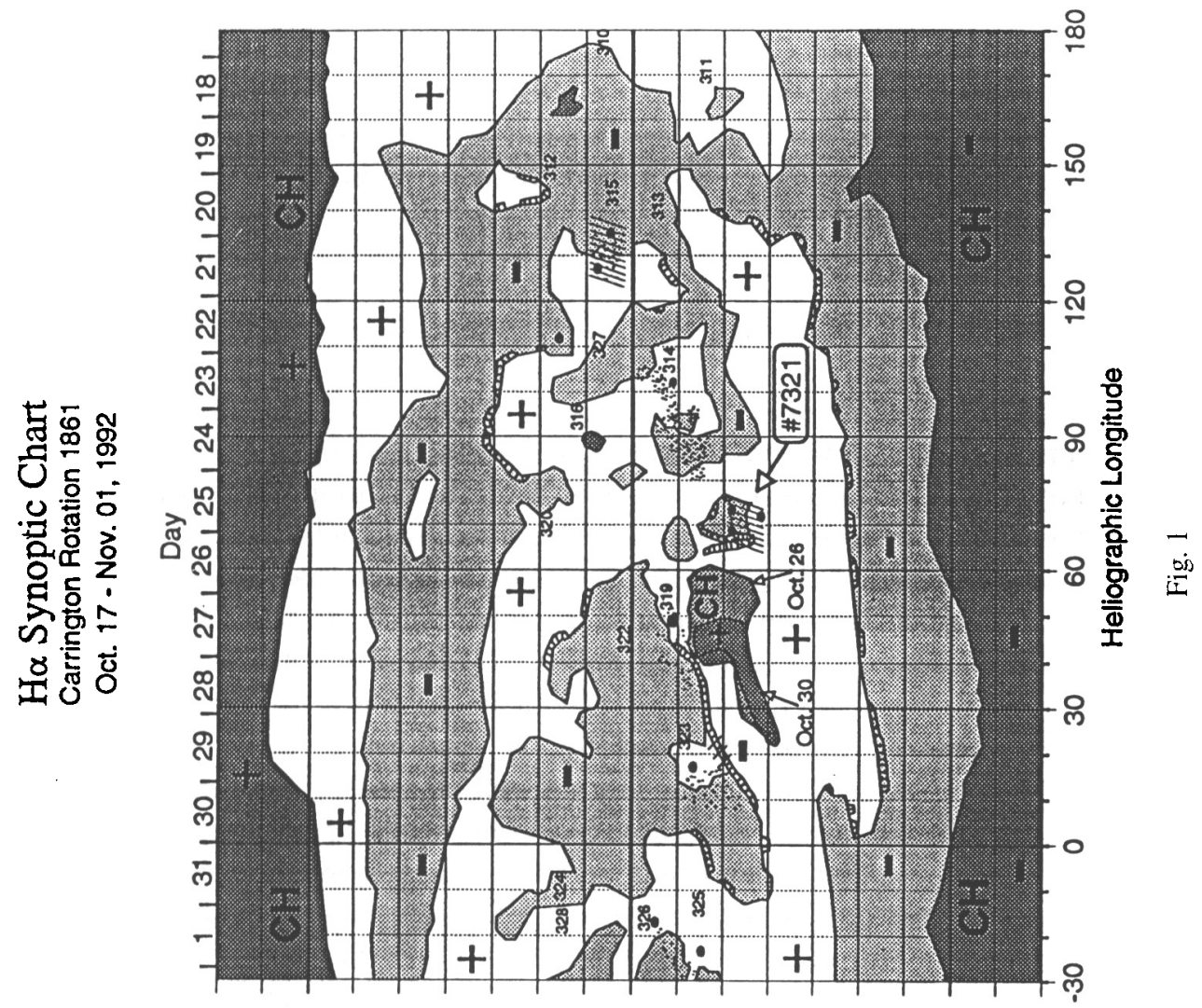

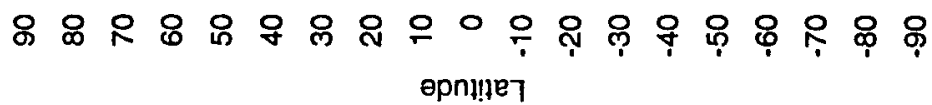


whether a coronal mass ejection (CME) occured or not. It is interesting to note that Storm Sudden Commencements (SSC) were also recorded on the Earth on November 1 and November 4, 1992, presumably corresponding to the above solar events, but the differences in the estimated and observed time interval between the trailing edge of the velocity fronts and the occurence of the SSC's were large.

\section{Summary and Discussion}

The above brief review of IPS observations for the study of interplanetary disturbances shows that solar events can be detected by the IPS method, particularly in a predictive mode. The IPS method suffers from integration along the line of sight to the radio source, but nevertheless can be used to detect the propagating disturbances by using a set of spatially distributed compact extragalactic radio sources as a 'picket fence'. In the present set of observations it was shown that the ability to estimate solar wind velocity using model fitting of temporal spectra can be advantageously used to not only complement ' $\mathrm{g}$ ' maps produced by the Cambridge observations, but helps us to time the events on the sun as well as trace the disturbances to the Earth. Such 'timing' efforts show that one needs to be cautious about assigning any particular solar event, such as flares, coronal holes or coronal mass ejections, as the sole cause of IPD's. Besides, it is known that geo-effectiveness of any IPD is closely related to the orientation of the interplanetary magnetic field (Tsurutani et al., 1992). Hence much work remains to be done before the method can become effective in establishing a connection between solar events, IPD's and disturbances on the Earth's magnetic field.

\section{Acknowledgements}

The review reports the results of cooperative endeavours over the past three years between the author and Drs. P.K. Manoharan and V. Balasubramanian of Tata Institute of Fundamental Research, India and P. Janardhan of Physical Research Laboratory, India and Drs. H. Leinbach, T. Detman and M. Dryer from the Space Environment Laboratory (SEL), Boulder, USA. The author is indebted to each one of the above colleagues for their contributions. The author also thanks Dr. P.S.McIntosh of HAO, Boulder for providing the figure 1 .

\section{References}

Dryer, M. and Smart, D.F.: 1984, Adv. Space Res. 4, 291 
Janardhan, P. et al: 1995, Solar Physics, in press

Leinbach, H., Ananthakrishnan, S. and Detman, T.R.: 1994, NOAA Technical Memorandum ERL SEL-83,

Manoharan, P.K and Ananthakrishnan, S: 1990, Mon. Not. Roy. astr. Soc 244, 691

Manoharan, P.K., et al: 1995, Solar Physics 156, 377 